\title{
Optimal control of stimulated Raman adiabatic passage in a superconducting qudit
}

Wen Zheng ${ }^{1,2}$, Yu Zhang ${ }^{1,2}$, Yuqian Dong ${ }^{1}$, Jianwen $\mathrm{Xu}^{1}$, Zhimin Wang ${ }^{1}$, Xiaohan Wang ${ }^{1}$, Yong Li ${ }^{1}$, Dong Lan ${ }^{1}$, Jie Zhao ${ }^{1}$, Shaoxiong Li ${ }^{1}$, Xinsheng $\operatorname{Tan}\left(\mathbb{D i D}^{1 \otimes}\right.$ and Yang $\mathrm{Yu}^{1 凶}$

Stimulated Raman adiabatic passage (STIRAP) is a widely used protocol to realize high-fidelity and robust quantum control in various quantum systems. However, further application of this protocol in superconducting qubits is limited by population leakage caused by the only weak anharmonicity. Here, we introduce an optimally controlled shortcut-to-adiabatic (STA) technique to speedup the STIRAP protocol in a superconducting qudit. By modifying the shapes of the STIRAP pulses, we experimentally realize a fast ( $32 \mathrm{~ns})$ and high-fidelity $(0.996 \pm 0.005)$ quantum state transfer. In addition, we demonstrate that our protocol is robust against control parameter perturbations. Our stimulated Raman shortcut-to-adiabatic passage transition provides an efficient and practical approach for quantum information processing.

npj Quantum Information (2022)8:9; https://doi.org/10.1038/s41534-022-00521-7

\section{INTRODUCTION}

Adiabatic passage techniques have been widely used to achieve reliable quantum control in quantum information processing. Among these techniques, stimulated Raman adiabatic passage (STIRAP) has achieved great success in physics, chemistry and beyond, since it was introduced by Gaubatz et al. ${ }^{1-8}$. In recent years, this approach is applied to quantum computation and quantum communication in superconducting circuits ${ }^{9-15}$. In addition, high-fidelity state transfer has been demonstrated in communication systems with multiple quantum nodes ${ }^{16}$. In a STIRAP scheme, the system Hamiltonian evolves adiabatically, so that its evolutionary trajectory is insensitive to the loss channel and noise ${ }^{17-19}$. This feature makes the approach important for future implementations of deterministic multi-qubit entanglement in quantum communication or computing networks based on superconducting circuits.

In general, the implementation of STIRAP is constrained by the strict adiabatic condition $2,20-25$, which is usually not feasible for superconducting qubits due to their fast decoherence caused by strong coupling with the environment ${ }^{26-29}$. To make the STIRAP approach more practical, it is desirable to speed-up the adiabatic procedure to counter the decoherence effect. For adiabatic quantum processes including STIRAP, in addition to combining composite pulses to enhance robustness while ensuring high fidelity ${ }^{30-35}$, various shortcut-to-adiabatic (STA) protocols have been theoretically and experimentally studied ${ }^{36-38}$, including counter-diabatic (CD) driving 23-25,39-41, invariants and scaling laws $^{42-45}$, variational methods ${ }^{46,47}$, and fast forward ${ }^{48,49}$. However, introducing these STA protocols in STIRAP (known as STIRSAP) may not be a good solution in transmon qubits due to weak anharmonicity. For instance, with CD driving, to suppress the nonadiabatic excitation from eigenstates, a coupling between the initial state and the target state is introduced ${ }^{11,12}$. However, this modification to the Hamiltonian will lead to unwanted population leakage in the adiabatic procedure ${ }^{26}$, prohibiting further improvement of transfer fidelity. It is possible to optimize the parameters of the driving microwave to suppress unwanted transitions for two-level systems. One can also use the derivative removal by adiabatic gate (DRAG) approach ${ }^{50,51}$ in a single qubit gate, but this procedure is not suitable for general three-level systems.

In this article, we improve the original STIRAP approach by experimentally demonstrating high-fidelity quantum transfer using STIRAP with optimized CD driving. By mapping the threelevel system to a two-level system, we add an additional CD driving term to the original Hamiltonian. We can then neglect the other dynamical coupling terms $\mathrm{s}^{52-54}$. Moreover, considering the effect of driving pulses on the transmon energy levels, we adopt the covariance matrix adaptation evolution strategy (CMA-ES) ${ }^{55}$ algorithm, which is a derivative-free evolution strategy, to optimize the leakage of the population on a non-computational basis. Our protocol provides a robust, high-fidelity method for fast quantum state transition.

\section{RESULTS}

\section{Shortcut-to-adiabatic approach}

Firstly, we consider the standard STIRAP approach, in which the Hamiltonian is written as $H=\frac{1}{2}\left[\Omega_{p}(t)|0\rangle\left\langle 1\left|+\Omega_{s}(t) e^{-i \phi}\right| 1\right\rangle\langle 2|+\right.$ h.c. $]$ ( $\hbar$ is set as 1). Its eigenstates include a dark state $|D\rangle=\cos (\theta)|0\rangle-$ $\sin (\theta) e^{-i \phi}|2\rangle$ and two bright states $\left|B_{1}\right\rangle=\frac{1}{\sqrt{2}}[\sin (\theta)|0\rangle+$ $\left.\cos (\theta) e^{-i \phi}|2\rangle+|1\rangle\right]$ and $\left|B_{2}\right\rangle=\frac{1}{\sqrt{2}}\left[\sin (\theta)|0\rangle+\cos (\theta) e^{-i \phi}|2\rangle+|1\rangle\right]$, where $\theta=\arctan \frac{\Omega_{p}(t)}{\Omega_{s}(t)}$ and $\phi$ is the relative phase between the P-and S-pulses. Without loss of generality, we set $\phi=0$ in this experiment. $\Omega_{p}(t)\left(\Omega_{s}(t)\right)$ is the envelope of the P-pulse (S-pulse) that drives the transition between $|0\rangle(|1\rangle)$ state and $|1\rangle(|2\rangle)$ state, as shown in the orange zone in Fig. 1a.

In our experiment, we use the Gaussian pulses

$$
\begin{aligned}
& \Omega_{p}(t)=\Omega_{0} e^{-(t-T / 2+\delta \tau)^{2} / \sigma^{2}} \\
& \Omega_{s}(t)=\Omega_{0} e^{-(t-T / 2-\delta \tau)^{2} / \sigma^{2}}
\end{aligned}
$$

as the P- and S-pulses, respectively, as denoted in Fig. 1b, where $\Omega_{0}$ is the Gaussian pulse amplitude, $T$ is the total evolution time, $\delta \tau=T / 11$ is the separation time between the two pulses, and $2 \sigma$

\footnotetext{
${ }^{1}$ National Laboratory of Solid State Microstructures, School of Physics, Nanjing University, 210093 Nanjing, China. ${ }^{2}$ These authors contributed equally: Wen Zheng, Yu Zhang.

凶email: tanxs@nju.edu.cn; yuyang@nju.edu.cn
} 
a
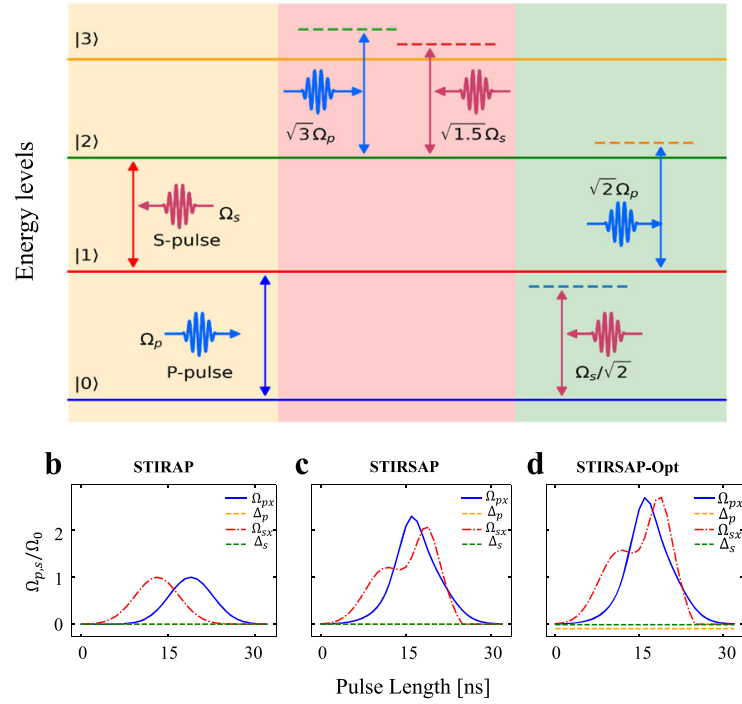

d STIRSAP-Opt

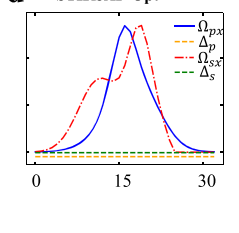

e

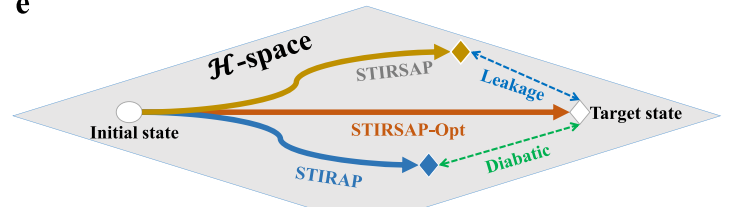

Fig. 1 Experimental schematic diagram. a Level diagram of the transmon driven by two external pulses with time-dependent amplitudes. In the first (orange) zone, the red (blue) pulse is the resonant pulse between $|0\rangle(|1\rangle)$ and $|1\rangle(|2\rangle)$, which is called the P-pulse (S-pulse). In the second (red) and third (green) zones, the red (blue) pulse is spurious coupling to other levels due to weak anharmonicity in the transmon. b-e Overview of different passages. As shown in e, in a multi-level system with weak anharmonicity, the conventional STIRAP (blue line) and STIRSAP (orange line) cannot achieve high-fidelity state transfer control due to the diabatic process and leakage when the evolution time is shortened, while STIRSAP-Opt (red line) can realize high fidelity. The envelopes of the three pulses from $\mathbf{b}$ to $\mathbf{d}$ are STIRAP, STIRSAP, and STIRSAP-Opt.

$=T / 6$ is the full-width at half-maximum of the pulse. To accelerate this STIRAP procedure while maintaining high fidelity, we modify the original $C D$ driving approach in two steps. First, we reconstruct the $C D$ driving term to avoid a two-photon transition. Second, we optimize the driving parameters using the CMA-ES routine. The Hamiltonian of the CD driving term is written as $H_{c d}=-i \Omega_{c d}|0\rangle\langle 2|+h . c$. , where $\Omega_{c d}(t)=\frac{\dot{\Omega}_{p}(t) \Omega_{s}(t)-\Omega_{p}(t) \dot{\Omega}_{s}(t)}{\Omega_{p}^{2}(t)+\Omega_{s}^{2}(t)}$. This term introduces the two-photon transition between states $|0\rangle$ and $|2\rangle$ in the transmon, which is troublesome for our routine. To simplify this problem, we modify the additional term in a new frame (see Methods) to cancel the two-photon transition, creating the coherent control pulse transform as below:

$$
\begin{gathered}
\tilde{\Omega}_{p}(t)=\Omega_{p}(t)-2 \dot{\zeta}(t) \\
\tilde{\Omega}_{s}(t)=\sqrt{\Omega_{p}(t)+4 \Omega_{c d}^{2}(t)},
\end{gathered}
$$

where $\zeta(t)=\arctan \frac{2 \Omega_{c d}(t)}{\Omega_{p}(t)}$.

\section{Optimization of driving parameters}

Now the system Hamiltonian with the STA approach becomes

$$
\begin{aligned}
H= & \sum_{n} \omega_{n}|n\rangle\langle n| \\
& +\sum_{k=p, s} \sum_{j}\left[\tilde{\Omega}_{k}(t) \cos \left(\omega_{k} t+\phi_{k}\right) \sqrt{j}|j-1\rangle\langle j|+\text { h.c. }\right]
\end{aligned}
$$

where index $j=1,2,3, n=0,1,2,3$ (considering the lowest four levels in the transmon) are the labels of energy eigenstates, $\phi_{k}$ is the initial phase of the P-pulse $(k=p)$ or the S-pulse $(k=s)$, and $\omega_{p}$ $\left(\omega_{s}\right)$ is the P-pulse (S-pulse) frequency. Notice that they can also drive other energy level transitions with certain detuning, which depends on the specific parameters of the transmon. Therefore, the problem we are dealing with is similar to the two-level system population leakage caused by weak anharmonicity, where we have to consider the effect of the spurious coupling terms and the Stark shift of the energy levels in the manipulation. However, the situation here is more complicated due to more energy levels being involved.

Here, we introduce the CMA-ES into STIRSAP to optimize the driving pulses and achieve high-fidelity state transfer control, which we call STIRSAP-Opt. For simplicity, we mainly improve the fidelity by optimizing the amplitudes and detunings of driving pulses. The driving pulses change from Fig. 1c, d, which can be written as

$$
\begin{array}{ccc}
\Omega_{p}(t)=a_{p} \tilde{\Omega}_{p}(t), & \Delta_{p}(t)=\beta_{p} \\
\Omega_{s}(t)=a_{s} \tilde{\Omega}_{s}(t), & \Delta_{s}(t)=\beta_{s}
\end{array}
$$

where $a_{p}\left(a_{s}\right)$ and $\beta_{p}\left(\beta_{s}\right)$ represent the amplitude coefficient and detuning of the P-pulse (S-pulse) to be optimized, respectively. We select a section in the parameter space (the amplitudes and the detunings form a four-dimensional parameter space in optimization) to visualize the optimization process. After the initial parameters are set, new candidate solutions (the black solid points) are generated by variations around the initial point, and the dotted line shows the distribution of the dots, as depicted in Fig. 2a.

In order to optimize parameters using CMA-ES, one can introduce the fidelity of state transfer control ${ }^{56}$

$\mathcal{F}=\operatorname{Tr} \sqrt{\sqrt{\rho_{\text {ideal }}} \rho_{\text {exp }} \sqrt{\rho_{\text {ideal }}}}$

and have a cost function

$\mathcal{C}=1-\mathcal{F}$.

Here, $\rho_{\text {exp }}$ is the qudit density matrix at $t=T$, and $\rho_{\text {ideal }}$ is the target state we want. In every iteration of the CMA-ES based on (6), some dots (individuals) are eliminated, and some dots are selected to become the new candidates (parents) in the next generation. Figure $2 b$ shows the intermediate generation, and in Fig. 2c, the optimization converges and gives a global optimal parameter set which will be used in STIRSAP-Opt. Given our experimental conditions, to show that STIRSAP-Opt can also achieve high fidelity in a very short period of time, we set $T=$ $32 \mathrm{~ns}$ and Rabi amplitude $\Omega_{0}=30 \mathrm{MHz}$ to demonstrate our routine. According to Eq. (1), Eq. (2), and Eq. (4), we get the driving pulses of three passages, shown in Fig. $1 \mathrm{~b}-\mathrm{d}$. Figure $2 \mathrm{~d}$, e show the state transfer process following STIRSAP and STIRSAPOpt. In these two cases, judging by the fidelity of state transfer and reduction of the population of the intermediate state $|1\rangle$, we find that STIRSAP-Opt has better performance. It is worth specifying that due to thermal excitation, the residue of state $|1\rangle$ exists during the entire procedure (More details see Supplementary Note 1). Within the short transfer time (32 ns), the STIRSAP performance is mainly limited by the leakage on a non-computational basis, whose fidelity is $0.900 \pm 0.006$. STIRSAPOpt, however, can speed-up the passage with rigorous leakage suppression with a fidelity of $0.996 \pm 0.005$ (In this article, the error bars indicate the $95 \%$ confidence interval). Meanwhile, based on Eq. (3), we also get the evolving population of states over time and calculate the corresponding fidelities are 0.919 and 0.999 for STIRSAP and STIRSAP-Opt, respectively, and they are in good agreement with the experimental results.

Next, we further study the speed-up of STIRSAP-Opt compared with STIRAP and STIRSAP. Without loss of generality, Rabi 

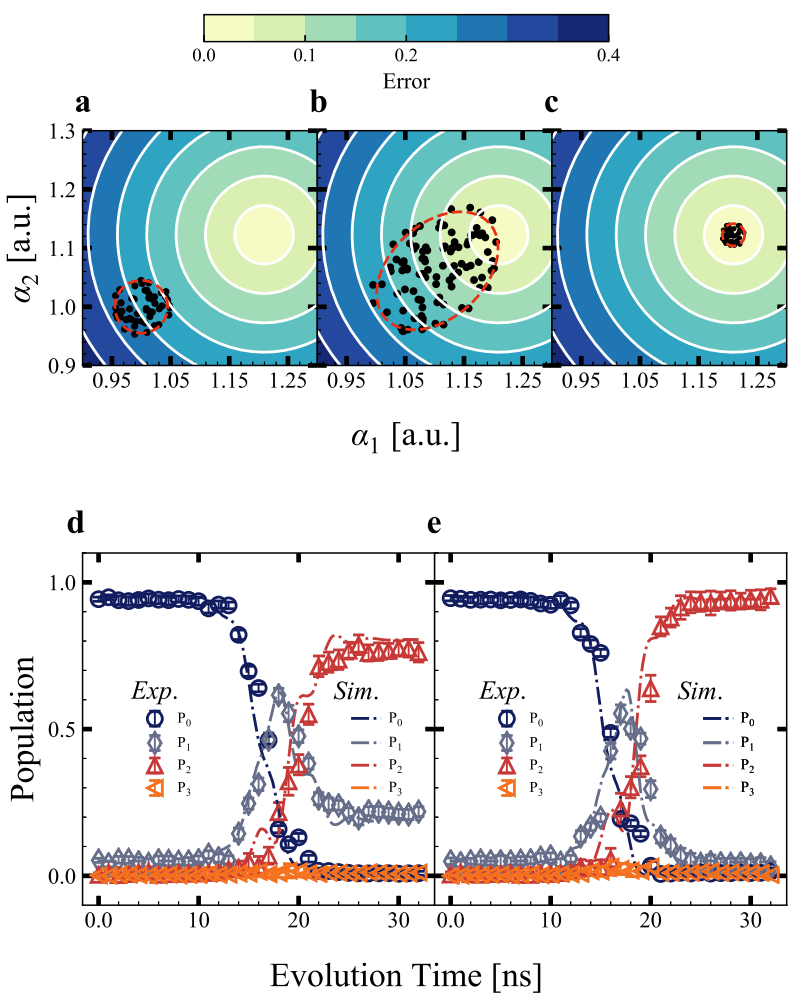

Fig. 2 State transfer control. a-c The CMA-ES-based optimization process in the amplitude subspace of the parameter space. a Initial parameters. b Intermediate generation parameters. c Final optimal parameters. d-e State transfer control simulation (line data) and experiment results (point data) with $32 \mathrm{~ns}$ manipulation time. d Transfer the initial state (state $|0\rangle$ ) to the target state (state $|2\rangle$ ) by STIRSAP. The fidelity of transfer control is $0.900 \pm 0.006$ and the fidelity in simulation is 0.919 . e Transfer the initial state (state $|0\rangle$ ) to the target state (state $|2\rangle$ ) by STIRSAP-Opt. The fidelity of transfer control is $0.996 \pm 0.005$, while it is 0.999 in simulation.

amplitude $\Omega_{0}$ is fixed as $20 \mathrm{MHz}$. We perform STIRAP, STIRSAP, and STIRSAP-Opt with different procedure times $T$, with the respective fidelities shown in Fig. 3c. The green horizontal dotted line indicates the threshold of fidelity at 0.99. Obviously, the shorter the time, the larger the advantage of our approach. Here, we emphasize two extreme conditions, which are $T=50 \mathrm{~ns}$ and $T=$ $500 \mathrm{~ns}$ (as vertical dotted line denoted in Fig. 3c). When $T=$ $500 \mathrm{~ns}$, the performance of all approaches is good, and the fidelities of STIRAP, STIRSAP, and STIRSAP-Opt are $0.982 \pm 0.013$, $0.984 \pm 0.011$, and $0.986 \pm 0.020$, while the simulation results are $0.981,0.980$, and 0.981 , respectively. At $T=50 \mathrm{~ns}$, the fidelities become $0.276 \pm 0.017,0.935 \pm 0.018$, and $0.994 \pm 0.021$, and the results in simulation are $0.179,0.932$, and 0.998 , respectively, proving that our method has significant advantage. We also compare the population of $|2\rangle$ in the three passages in Fig. 3a, b, and the results agree with the fidelity results. Here, we point out that the errors at $\mathrm{T}=500 \mathrm{~ns}$ mainly come from the influences of decoherence. The performance of STIRSAP-Opt without decoherence sees Supplementary Note 2.

Furthermore, we have experimentally verified that the state transfer control based on STIRSAP-Opt has impressive robustness while maintaining high fidelity. As shown in Fig. 4a, c, we measured the fidelity by changing the P- and S-pulses amplitude and frequency, which are similar to errors of driving pulses in the experiment. As shown in Fig. 2e, the length of the driving pulses we use is $32 \mathrm{~ns}$, and we define driving amplitude error $\eta_{k}=1-$ $\Omega_{k} / \Omega_{\text {ref, }, k}$ and driving frequency error $\delta_{k}=\Delta_{k}-\Delta_{\text {ref, } k}$ where the index $k=p, s$ means the P- and S-pulses, and $\Omega_{\text {ref, } k}$ and $\Delta_{r e f, k}$ are

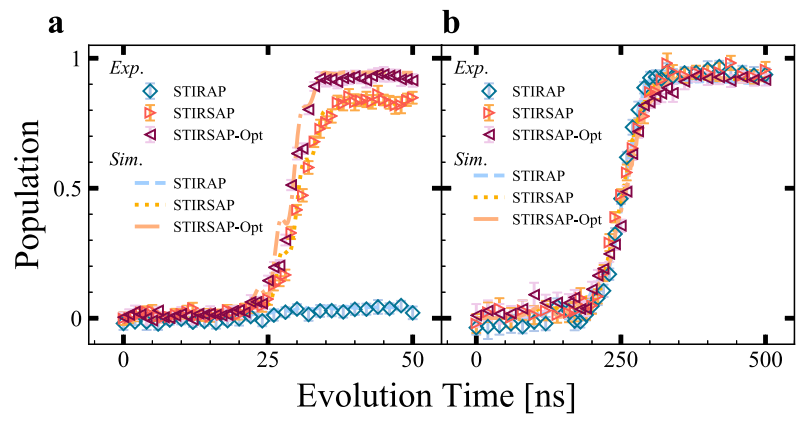

c

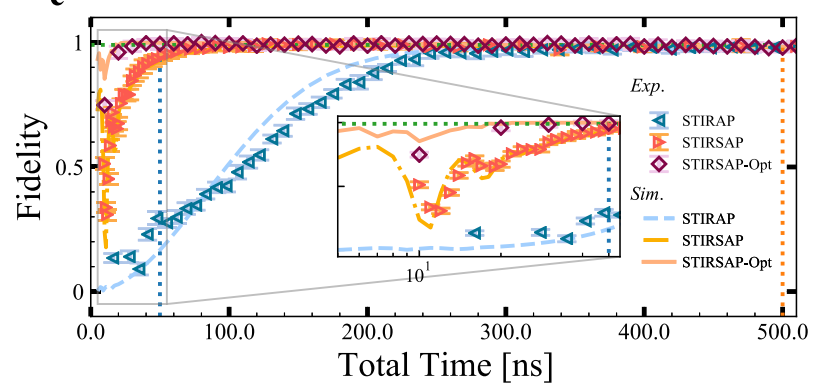

Fig. 3 The fidelity of state transfer control with evolution total time. a The evolution of the target state $(|2\rangle$ states) population is within $50 \mathrm{~ns}$ (here, $T=T_{0}, T_{0}=2 \pi / \Omega_{0}$ and $\Omega_{0}=2 \pi \times 20 \mathrm{MHz}$ ). The fidelities of transfer control are $0.276 \pm 0.017,0.935 \pm 0.018,0.994 \pm$ 0.021 for STIRAP, STIRSAP, STIRSAP-Opt, respectively, and in simulation, they are $0.179,0.932,0.998$. For $T=10 T_{0}$ in $\mathbf{b}$, however, it means that the conventional adiabatic approximation condition is satisfied. The fidelities of transfer control are $0.982 \pm 0.013,0.984 \pm$ $0.011,0.986 \pm 0.020$, respectively. The fidelities in simulation are $0.981,0.980,0.981$, respectively. c Measured (point data) and simulated (line data) fidelity of transfer control as a function of the total time with reference driving amplitude $\Omega_{0}$ using STIRAP, STIRSAP, and STIRSAP-Opt, respectively. The two dotted lines at 50 and 500 ns mark the moments when the total time is the time $T$ in a and $\mathbf{b}$, respectively. The inset image zooms in the part of $T \in[5,55]$ ns to more intuitively compare the differences between STIRAP, STIRSAP, and STIRSAP-Opt.

optimal amplitude and detuning by STIRSAP-Opt, respectively. Meanwhile, we use QuTiP ${ }^{57,58}$ to simulate the process under the experimental conditions. The results are shown in Fig. $4 \mathrm{~b}, \mathrm{~d}$. In the experiment, the amplitude-frequency response of the experimental circuit is nonlinear under different amplitudes and frequencies. For short pulses, the effect of these errors may be especially serious due to leakage caused by the weak anharmonicity, leading to the differences between the experiment and the simulation. In order to highlight the details of the differences, we have selected data along with the antidiagonal of experimental results (Fig. 4a, C) and simulation results (Fig. $4 \mathrm{~b}, \mathrm{~d}$ ) to plot Fig. 4e, f. Here, although the pulses are short ( $32 \mathrm{~ns})$, our experimental results and simulation results are in good agreement.

\section{DISCUSSION}

In conclusion, we eliminated the two-photon resonance channel to reduce the complexity of manipulation after introducing $C D$ driving into the STIRAP protocol under resonance conditions in superconducting circuits. Considering the weak anharmonicity in the transmon qudit, we combined the optimization algorithm CMA-ES to achieve fast ( $32 \mathrm{~ns})$ and high-fidelity $(0.996 \pm 0.005)$ state transfer. Our method can be directly applied to quantum manipulation in quantum communication, especially for systems that are composed of multiple qubits or multi-quantum nodes, which have the loss channel or spontaneous emission of the intermediate state. Thus, quantum manipulation with high fidelity 


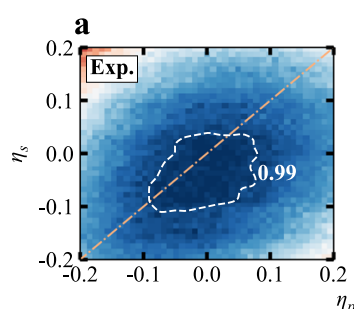

b
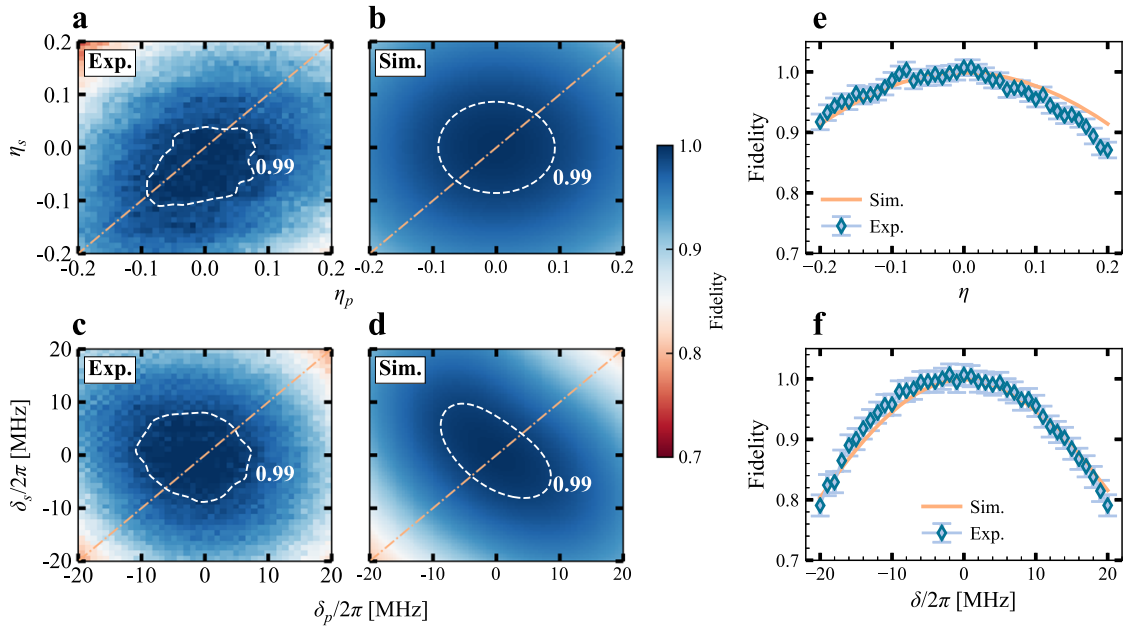

Fig. 4 Robustness. $\mathbf{a}$ and $\mathbf{c}$ are the robustness of state transfer control against errors in the driving pulse amplitude and detuning for STIRSAPOpt, respectively, and $\mathbf{b}$ and $\mathbf{d}$ are respective theoretical simulations. $\mathbf{e}$ and $\mathbf{f}$ are details of the transfer fidelity along the antidiagonal of $\mathbf{a}$ and c, respectively. The points (lines) are experimental (simulation) results.

and fast speed can be realized with intermediate coherent times and under experimental conditions.

\section{METHODS}

\section{Theoretical model}

Based on the description in the text, considering a three-level system and after introducing $C D$ driving, we have the Hamiltonian

$$
H=\frac{1}{2}\left(\begin{array}{ccc}
0 & \Omega_{p}(t) & \Omega_{c d}(t) \\
\Omega_{p}(t) & 0 & \Omega_{s}(t) \\
\Omega_{c d}(t) & \Omega_{s}(t) & 0
\end{array}\right)
$$

where

$$
\Omega_{c d}(t)=\frac{\dot{\Omega}_{p}(t) \Omega_{s}(t)-\Omega_{p}(t) \dot{\Omega}_{s}(t)}{\Omega_{p}^{2}(t)+\Omega_{s}^{2}(t)}
$$

is the $C D$ driving term to suppress the non-adiabatic emission in evolution. The Hamiltonian itself satisfies the intrinsic SU(2) Lie algebra ${ }^{52}$. Using the Gell-Mann matrices, we have

$H=\frac{1}{2}\left[\Omega_{p}(t) \lambda_{1}+\Omega_{s}(t) \lambda_{6}-2 \Omega_{c d}(t) \lambda_{5}\right]$,

where

$$
\lambda_{1}=\left(\begin{array}{lll}
0 & 1 & 0 \\
1 & 0 & 0 \\
0 & 0 & 0
\end{array}\right), \lambda_{5}=\left(\begin{array}{ccc}
0 & 0 & -i \\
0 & 0 & 0 \\
i & 0 & 0
\end{array}\right), \lambda_{6}=\left(\begin{array}{lll}
0 & 0 & 0 \\
0 & 0 & 1 \\
0 & 1 & 0
\end{array}\right) .
$$

Therefore, when $U(t)=e^{-i \zeta(t) \lambda_{6}}$ is introduced for unitary transformation, we can obtain the Hamiltonian

$H=\frac{1}{2}\left[\tilde{\Omega}_{p}(t) \lambda_{1}+\tilde{\Omega}_{s}(t) \lambda_{6}-2 \tilde{\Omega}_{c d}(t) \lambda_{5}\right]$

Suppose $\tilde{\Omega}_{c d}=0$, we get $\zeta(t)$. Under this condition, the initial state and the final state are not directly coupled, and we can get the coherent control pulses based on Eq. (2). When the evolution time becomes short, the second-order rotating wave approximation fails in systems with weak anharmonicity, and the Hamiltonian becomes Eq. (3). We consider four levels and define a unitary matrix $U=\sum_{n} e^{-i \theta_{n}}|n\rangle\langle n|$ (where $\theta_{n}=\omega_{n} t$ and $\omega_{p}\left(\omega_{s}\right)$ is the P-pulse (S-pulse) driving frequency). We have

$$
\begin{aligned}
H= & \frac{1}{2}\left[\tilde{\Omega}_{p}(t)+\frac{1}{\sqrt{2}} \tilde{\Omega}_{s}(t) e^{-i \delta_{p}}|0\rangle\langle 1|\right. \\
& \left.+\sqrt{2} \tilde{\Omega}_{p}(t) e^{i \delta_{s}}+\tilde{\Omega}_{s}(t)\right]|1\rangle\langle 2| \\
& \left.\left.+\sqrt{3} \tilde{\Omega}_{p}(t) e^{i \delta_{1}}+\sqrt{\frac{3}{2}} \tilde{\Omega}_{s}(t) e^{i \delta_{2}}\right]|2\rangle\langle 3|+\text { h.c. }\right]
\end{aligned}
$$

We set $\omega_{0}=0 \mathrm{GHz}$, and $\delta_{p}=\left(\omega_{1}-\omega_{p}\right) t+\phi_{p}, \delta_{s}=\left(\omega_{2}-\omega_{1}-\omega_{s}\right) t+\phi_{s} \delta_{1}$ $=\left(\omega_{1}-\omega_{s}\right) t+\phi_{s t}$ and $\delta_{2}=\left(\omega_{2}-\omega_{1}-\omega_{p}\right) t+\phi_{p}$. As shown in the formula above and in Fig. 1a, the fidelity of state transfer is low because there are coupling terms between other unnecessary energy levels.

\section{DATA AVAILABILITY}

Data that support the findings of this study are available from the corresponding author upon reasonable request.

\section{CODE AVAILABILITY}

The codes developed for the simulations of this study are available from the corresponding author upon reasonable request.

Received: 9 July 2021; Accepted: 15 December 2021; Published online: 27 January 2022

\section{REFERENCES}

1. Gaubatz, U. et al. Population switching between vibrational levels in molecular beams. Chem. Phys. Lett. 149, 463-468 (1988).

2. Gaubatz, U., Rudecki, P., Schiemann, S. \& Bergmann, K. Population transfer between molecular vibrational levels by stimulated raman scattering with partially overlapping laser fields. a new concept and experimental results. J. Chem. Phys. 92, 5363-5376 (1990).

3. Vitanov, N., Halfmann, T., Shore, B. \& Bergmann, K. Laser-induced population transfer by adiabatic passage techniques. Ann. Rev. Phys. Chem. 52, 763-809 (2001).

4. Král, P., Thanopulos, I. \& Shapiro, M. Colloquium: coherently controlled adiabatic passage. Rev. Mod. Phys. 79, 53-77 (2007).

5. Shore, B. W. Coherent manipulations of atoms using laser light. Acta Phys. Slovaca. Rev. Tutor. 58, 243-486 (2008).

6. Shore, B. W. Pre-history of the concepts underlying stimulated Raman adiabatic passage (stirap). Acta Phys. Slovaca 63, 361-482 (2013).

7. Bergmann, K., Vitanov, N. V. \& Shore, B. W. Perspective: stimulated Raman adiabatic passage: The status after 25 years. J. Chem. Phys. 142, 170901 (2015).

8. Vitanov, N. V., Rangelov, A. A., Shore, B. W. \& Bergmann, K. Stimulated Raman adiabatic passage in physics, chemistry, and beyond. Rev. Mod. Phys. 89, 015006 (2017).

9. Kumar, K., Vepsäläinen, A., Danilin, S. \& Paraoanu, G. Stimulated Raman adiabatic passage in a three-level superconducting circuit. Nat. Commun. 7, 10628 (2016).

10. $\mathrm{Xu}, \mathrm{H}$. et al. Coherent population transfer between uncoupled or weakly coupled states in ladder-type superconducting qutrits. Nat. Commun. 7, 11018 (2016).

11. Vepsäläinen, A., Danilin, S. \& Paraoanu, G. S. Superadiabatic population transfer in a three-level superconducting circuit. Sci. Adv. 5, eaau5999 (2019).

12. Yang, Z. et al. Realization of arbitrary state-transfer via superadiabatic passages in a superconducting circuit. Appl. Phys. Lett. 115, 072603 (2019)

13. Niu, J. et al. Quantum control via stimulated Raman user-defined passage. Preprint at https://arxiv.org/abs/1912.10927 (2020). 
14. Liu, B.-J. \& Yung, M.-H. Coherent control with user-defined passage. Quant. Sci. Technol. 6, 025002 (2021).

15. Li, D. et al. Coherent state transfer between superconducting qubits via stimulated Raman adiabatic passage. Appl. Phys. Lett. 118, 104003 (2021).

16. Chang, H.-S. et al. Remote entanglement via adiabatic passage using a tunably dissipative quantum communication system. Phys. Rev. Lett. 124, 240502 (2020)

17. Yatsenko, L. P., Shore, B. W. \& Bergmann, K. Detrimental consequences of small rapid laser fluctuations on stimulated Raman adiabatic passage. Phys. Rev. A 89, 013831 (2014).

18. Blekos, K., Stefanatos, D. \& Paspalakis, E. Performance of superadiabatic stimulated Raman adiabatic passage in the presence of dissipation and Ornsteinuhlenbeck dephasing. Phys. Rev. A 102, 023715 (2020).

19. Hou, Q. Z., Yang, W. L., Feng, M. \& Chen, C.-Y. Quantum state transfer using stimulated Raman adiabatic passage under a dissipative environment. Phys. Rev. A 88, 013807 (2013).

20. Kato, T. On the adiabatic theorem of quantum mechanics. J. Phys. Soc. Japan $\mathbf{5}$, 435-439 (1950)

21. Kuklinski, J. R., Gaubatz, U., Hioe, F. T. \& Bergmann, K. Adiabatic population transfer in a three-level system driven by delayed laser pulses. Phys. Rev. A $\mathbf{4 0}$ 6741-6744 (1989).

22. Kuhn, A. et al. Population transfer by stimulated Raman scattering with delayed pulses using spectrally broad light. J. Chem. Phys. 96, 4215-4223 (1992).

23. Berry, M. V. Transitionless quantum driving. J. Phys. A: Math. Theor. 42, 365303 (2009).

24. Demirplak, M. \& Rice, S. A. On the consistency, extremal, and global properties of counterdiabatic fields. J. Chem. Phys. 129, 154111 (2008).

25. del Campo, A. Shortcuts to adiabaticity by counterdiabatic driving. Phys. Rev. Lett. 111, 100502 (2013)

26. Koch, J. et al. Charge-insensitive qubit design derived from the cooper pair box. Phys. Rev. A 76, 042319 (2007).

27. Xiang, Z.-L., Ashhab, S., You, J. Q. \& Nori, F. Hybrid quantum circuits: Superconducting circuits interacting with other quantum systems. Rev. Mod. Phys. 85, 623-653 (2013).

28. Gu, X., Kockum, A. F., Miranowicz, A., xi Liu, Y. \& Nori, F. Microwave photonics with superconducting quantum circuits. Phys. Rep. 718-719, 1-102 (2017).

29. Blais, A., Grimsmo, A. L., Girvin, S. M. \& Wallraff, A. Circuit quantum electrodynamics. Rev. Mod. Phys. 93, 025005 (2021).

30. Levitt, M. H. Composite pulses. Prog. Nucl. Magn. Reson. Spectrosc. 18, 61-122 (1986)

31. Genov, G. T., Schraft, D., Halfmann, T. \& Vitanov, N. V. Correction of arbitrary field errors in population inversion of quantum systems by universal composite pulses. Phys. Rev. Lett. 113, 043001 (2014).

32. Torosov, B. T. \& Vitanov, N. V. Composite stimulated raman adiabatic passage. Phys. Rev. A 87, 043418 (2013).

33. Bruns, A., Genov, G. T., Hain, M., Vitanov, N. V. \& Halfmann, T. Experimental demonstration of composite stimulated raman adiabatic passage. Phys. Rev. A 98, 053413 (2018).

34. Laforgue, X., Chen, X. \& Guérin, S. Robust stimulated raman exact passage using shaped pulses. Phys. Rev. A 100, 023415 (2019).

35. Shi, Z.-C. et al. Robust single-qubit gates by composite pulses in three-level systems. Phys. Rev. A 103, 052612 (2021).

36. Emmanouilidou, A., Zhao, X.-G., Ao, P. \& Niu, Q. Steering an eigenstate to a destination. Phys. Rev. Lett. 85, 1626-1629 (2000).

37. Torrontegui, E. et al. In Advances in Atomic, Molecular, and Optical Physics, Vol. 62 (eds Arimondo, E., Berman, P. R. \& Lin, C. C.) 117-169 (Academic Press, 2013).

38. Guéry-Odelin, D. et al. Shortcuts to adiabaticity: Concepts, methods, and applications. Rev. Mod. Phys. 91, 045001 (2019)

39. Unanyan, R., Yatsenko, L., Bergmann, K. \& Shore, B. Laser-induced adiabatic atomic reorientation with control of diabatic losses. Opt. Commun. 139, 48-54 (1997).

40. Fleischhauer, M., Unanyan, R., Shore, B. W. \& Bergmann, K. Coherent population transfer beyond the adiabatic limit: generalized matched pulses and higher-order trapping states. Phys. Rev. A 59, 3751-3760 (1999).

41. Petiziol, F., Arimondo, E., Giannelli, L., Mintert, F. \& Wimberger, S. Optimized threelevel quantum transfers based on frequency-modulated optical excitations. Sci. Rep. 10, 2185 (2020).

42. Xu, T.-N., Liu, K., Chen, X. \& Guérin, S. Invariant-based optimal composite stimulated raman exact passage. J. Phys. B: Atom. Mol. Opt. Phys. 52, 235501 (2019).

43. Feng, Z.-B. \& Lu, X.-J. Optimal controls of invariant-based population transfer in a superconducting qutrit. Quantum Inf. Process. 19, 83 (2020).

44. Han, Z. et al. Realization of invariant-based shortcuts to population inversion with a superconducting circuit. Appl. Phys. Lett. 118, 224003 (2021).

45. Song, X.-K. et al. Robust stimulated Raman shortcut-to-adiabatic passage with invariant-based optimal control. Opt. Express 29, 7998 (2021).

46. Kolodrubetz, M., Sels, D., Mehta, P. \& Polkovnikov, A. Geometry and non-adiabatic response in quantum and classical systems. Phys. Rep. 697, 1-87 (2017).

47. Sels, D. \& Polkovnikov, A. Minimizing irreversible losses in quantum systems by local counterdiabatic driving. Proc. Natl Acad. Sci. 114, E3909-E3916 (2017).
48. Lewis, H. R. \& Riesenfeld, W. B. An exact quantum theory of the time-dependent harmonic oscillator and of a charged particle in a time-dependent electromagnetic field. J. Math. Phys. 10, 1458-1473 (1969).

49. Chen, X. et al. Fast optimal frictionless atom cooling in harmonic traps: Shortcut to adiabaticity. Phys. Rev. Lett. 104, 063002 (2010)

50. Motzoi, F., Gambetta, J. M., Rebentrost, P. \& Wilhelm, F. K. Simple pulses for elimination of leakage in weakly nonlinear qubits. Phys. Rev. Lett. 103, 110501 (2009).

51. Gambetta, J. M., Motzoi, F., Merkel, S. T. \& Wilhelm, F. K. Analytic control methods for high-fidelity unitary operations in a weakly nonlinear oscillator. Phys. Rev. A 83, 012308 (2011).

52. Li, Y.-C. \& Chen, X. Shortcut to adiabatic population transfer in quantum threelevel systems: effective two-level problems and feasible counterdiabatic driving. Phys. Rev. A 94, 063411 (2016).

53. Du, Y.-X. et al. Experimental realization of stimulated Raman shortcut-to-adiabatic passage with cold atoms. Nat. Commun. 7, 12479 (2016).

54. Baksic, A., Ribeiro, H. \& Clerk, A. A. Speeding up adiabatic quantum state transfer by using dressed states. Phys. Rev. Lett. 116, 230503 (2016).

55. Hansen, N. The CMA evolution strategy: a tutorial. Preprint at https://arxiv.org/ abs/1604.00772 (2016)

56. Nielsen, M. A. \& Chuang, I. L.Quantum Computation and Quantum Information: 10th Anniversary Edition (Cambridge University Press, 2010).

57. Johansson, J., Nation, P. \& Nori, F. Qutip: An open-source python framework for the dynamics of open quantum systems. Comput. Phys. Commun. 183, 1760-1772 (2012).

58. Johansson, J., Nation, P. \& Nori, F. Qutip 2: A python framework for the dynamics of open quantum systems. Comput. Phys. Communications 184, 1234-1240 (2013).

\section{ACKNOWLEDGEMENTS}

This work was supported by the Key R\&D Program of Guangdong Province (Grant No. 2018B030326001), NSFC (Grants No. 11890704, No. 12074179 and No. 61521001)).

\section{AUTHOR CONTRIBUTIONS}

X.T. and W.Z. conceived the experiments; W.Z. carried out the measurements with the help of Y.Z. and Y.L.; Y.Z. and W.Z. analyzed the data with the help of X.T. and Y.Y.; J.X. and D.L. prepared the sample; Y.Z. and W.Z. performed the numerical calculations; W. Z., Y.Z., X.T., and Y.Y. wrote the paper. All authors discussed the results.

\section{COMPETING INTERESTS}

The authors declare no competing interests.

\section{ADDITIONAL INFORMATION}

Supplementary information The online version contains supplementary material available at https://doi.org/10.1038/s41534-022-00521-7.

Correspondence and requests for materials should be addressed to Xinsheng Tan or Yang Yu.

Reprints and permission information is available at http://www.nature.com/ reprints

Publisher's note Springer Nature remains neutral with regard to jurisdictional claims in published maps and institutional affiliations.

Open Access This article is licensed under a Creative Commons Attribution 4.0 International License, which permits use, sharing, adaptation, distribution and reproduction in any medium or format, as long as you give appropriate credit to the original author(s) and the source, provide a link to the Creative Commons license, and indicate if changes were made. The images or other third party material in this article are included in the article's Creative Commons license, unless indicated otherwise in a credit line to the material. If material is not included in the article's Creative Commons license and your intended use is not permitted by statutory regulation or exceeds the permitted use, you will need to obtain permission directly from the copyright holder. To view a copy of this license, visit http://creativecommons. org/licenses/by/4.0/.

(c) The Author(s) 2022 